\title{
Retorno a la actividad asistencial en el contexto de COVID-19: priorización, protocolos y procedimientos. Experiencia de una unidad de gastroenterología ambulatoria en Bogotá, Colombia
}

\author{
Restarting care-related activities in the context of COVID-19: Prioritization, \\ protocols, and procedures. Experience of a gastroenterology outpatient unit in \\ Bogotá, Colombia
}

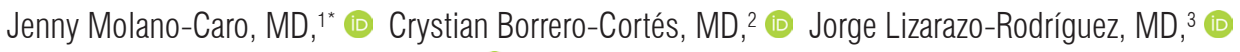

Néstor Eduardo Gómez-Chacón, MD. ${ }^{4}$ (1)

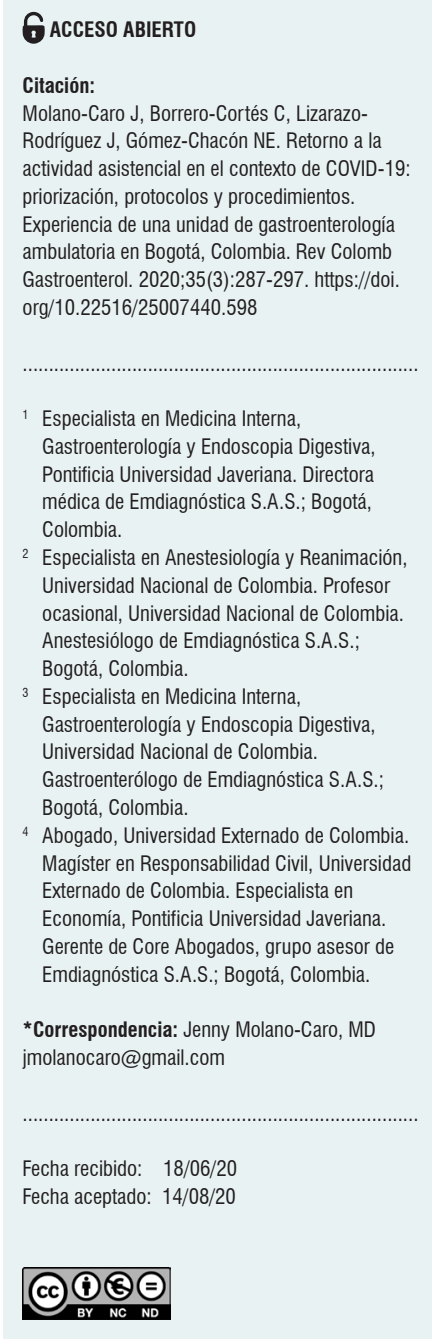

\section{Resumen}

La infección por el virus SARS-CoV-2 en Colombia condujo a que el Gobierno decretara una cuarentena total obligatoria en marzo de 2020. En dicho momento, las unidades de endoscopia ambulatoria permanecieron cerradas y solo se efectuaron procedimientos urgentes en el ámbito hospitalario. Esto ocasionó un represamiento de procedimientos endoscópicos sensibles al tiempo, prioritarios y electivos, de realización ambulatoria. La velocidad de propagación del virus se ha contenido, lográndose detener la progresión exponencial observada inicialmente. En dicho contexto, la atención de gastroenterología se ha continuado en la modalidad de teleconsulta. En mayo de este mismo año, el comportamiento de contención epidemiológica permitió que la Alcaldía de Bogotá y el Gobierno nacional emitieran circulares con las recomendaciones para la prestación de algunos servicios ambulatorios reglamentados, y así dar oportunidad de atención a pacientes con enfermedades sensibles al tiempo. Dentro de este marco legal y epidemiológico, se reinició la prestación de servicios de endoscopia digestiva en Emdiagnóstica S.A.S. Por tanto, presentamos aquí las estrategias para la programación y la realización de procedimientos endoscópicos en una unidad ambulatoria de gastroenterología, durante la pandemia por COVID-19 (Coronavirus disease 2019) en Colombia. También se describe un sistema de priorización de procedimientos de acuerdo con el criterio médico, que va desde la atención por teleconsulta o la aplicación de una encuesta telefónica hasta la utilización de una escala MeNTS (Medically Necessary Time-Sensitive) adaptada para endoscopia digestiva. Además, se relatan las adecuaciones en la planta física, la metodología implementada para la protección del talento humano y de los pacientes y el seguimiento posterior a la realización de los procedimientos para retroalimentación, seguridad y evaluación de satisfacción.

\section{Palabras clave}

SARS-CoV-2, COVID-19, endoscopia, calidad, seguridad, sedación.

\section{Abstract}

The Colombian Government ordered a mandatory nationwide quarantine in March 2020 due to the spread of the SARS-CoV-2 virus. Since then, outpatient endoscopy units were closed and only urgent procedures were performed in the hospital setting, resulting in a repression of sensitive, priority and elective outpatient endoscopic procedures. The rate of spread of the virus was contained and it did not progress exponentially as in other countries; in the meantime, gastroenterology services were provided in the form of teleconsultation. The mitigation measures and the containment of the virus allowed the Mayor's Office of Bogota and the National Government to issue notices with recommendations for the provision of some regulated outpatient services in May 2020, thus creating a window of opportunity to care for patients with sensitive diseases. Under this legal and epidemiological framework, the provision of digestive endoscopy services was restarted at EMDIAGNOSTICA S.A.S. This study presents the strategies for scheduling and performing endoscopic procedures in an outpatient gastroenterology unit during COVID-19 pandemia in Colombia and describes a system for prioritizing procedures according to medical criteria, ranging from care by teleconsultation and/ 
or an application of a telephone survey and the use of a medically necessary, time-sensitive (MENTS) scale adapted for digestive endoscopy. It also describes changes in infrastructure, methodology implemented for protection of human talent and patients, and post procedure follow-up for feedback, safety and satisfaction degree evaluation in care.

Keywords

SARS-CoV-2; COVID-19; Endoscopy; Quality; Safety; Sedation.

\section{INTRODUCCIÓN Y PLANTEAMIENTO DEL PROBLEMA}

La enfermedad por coronavirus (Coronavirus Disease 2019, COVID-19), declarada pandemia por la Organización Mundial de la Salud (OMS) el 11 de marzo de 2020 (1), modificó de manera sustancial la prestación de servicios de salud para la comunidad y el personal asistencial. En Colombia, se decretó cuarentena obligatoria en marzo de 2020, con el objetivo de retrasar el pico de infectados, lo cual permitió a la red hospitalaria generar la adaptación necesaria para no colapsar $(2,3)$. Desde el primer caso, reportado el 6 de marzo de ese año (4), se han establecido protocolos de aislamiento ajustados al fin de permitir los desplazamientos de personas y el retorno a las actividades laborales, pero considerando siempre como prioridad el mantenimiento de la seguridad de los ciudadanos $(5,6)$.

Los lineamientos gubernamentales fueron específicos en restringir la prestación de algunos servicios médicos no esenciales $(2,3)$. En particular, aquellos servicios involucrados con el diagnóstico de enfermedades sensibles al tiempo (en las que el momento en el cual se realizan las intervenciones diagnósticas o terapéuticas genera un efecto directo en su pronóstico) tuvieron que enfrentarse a cumplir con los decretos de cuarentena y el aislamiento preventivo, pero al mismo tiempo formular opciones a fin de permitir la realización de procedimientos diagnósticos indispensables en el pronóstico de algunas enfermedades, sin que esto representara un riesgo mayor para la salud.

$\mathrm{Al}$ considerarse que los procedimientos endoscópicos gastrointestinales son generadores (o potencialmente generadores) de aerosoles, se desarrollaron estrategias para disminuir el riesgo de transmisión durante su práctica. Luego de 7 semanas de cierre, las unidades ambulatorias pudieron reiniciar la prestación de sus servicios sin saber por cuánto tiempo. En consecuencia, definir a quiénes y cómo reiniciar la actividad en las unidades de endoscopia fue una pregunta obligada.

En nuestra unidad, una vez identificado el problema, comenzó a planearse una estrategia para afrontar la situación. Así, se conformó un grupo multidisciplinario (gastroenterólogos, anestesiólogos, abogados, enfermeras y asesores financieros) que, mediante reuniones virtuales, construyó un protocolo con recomendaciones a fin de reactivar la prestación de servicios, y basado en un esquema de priorización descrito a continuación.

\section{METODOLOGÍA}

Antes de la reapertura de la unidad, se realizó una búsqueda no sistemática en la literatura médica (Medline-Pubmed, Scielo, Embase y Google-Scholar) sobre protocolos, guías de práctica clínica y reportes en países que ya habían experimentado esta situación. Además, se participó en un importante número de conferencias virtuales y se revisaron los diferentes decretos emanados por entes gubernamentales $(2,3)$. Por fortuna, fue posible conocer las intervenciones realizadas internacionalmente, reproducir las acciones que mostraron ser efectivas y modificar o adaptar aquellas susceptibles de mejora.

Las diferentes asociaciones científicas internacionales (7-16) diseñaron y socializaron guías que fueron revisadas y adaptadas para la situación particular, dado que su aplicación debía ajustarse a las necesidades de una unidad de endoscopia ambulatoria que no presta servicios de urgencias. Todas estas medidas de contingencia se planearon de acuerdo con el comportamiento de la COVID-19 en Bogotá.

\section{MARCO LEGAL}

El plan de acción para la prestación de servicios de salud durante la contención y mitigación de la pandemia fue adoptado por el Ministerio de Salud, mediante la resolución 536 de 2020. Sobre ella se estructuraron los planes de atención en el contexto de la crisis. Es así como en abril y mayo se expidieron los lineamientos para la restauración gradual de los servicios en las fases de mitigación y control de la emergencia sanitaria. Se hizo énfasis en cirugía ambulatoria, cirugía con bajo riesgo de requerimiento de unidad de cuidados intensivos (UCI), cirugías de corta estancia hospitalaria y cirugías no diferibles.

En la actualización de junio, se eliminaron las recomendaciones respecto a los servicios de consulta externa, ciru- 
gía y procedimientos de apoyo diagnóstico, señalando que "se podrán prestar los servicios y realizar los procedimientos no diferibles, de forma tal, que se garantice la protección y seguridad de los usuarios y del talento humano asociado a la realización de las atenciones".

Asimismo, el Ministerio de Salud indicó que las acciones por seguir y la posibilidad de reactivación de los servicios depende del análisis territorial de factores como la tendencia de nuevos casos, la suficiencia del talento humano, la disponibilidad de elementos de protección personal (EPP), la utilización de la capacidad instalada y el comportamiento en la prestación del servicio. Por tanto, la implementación de cualquier plan de reactivación debe pasar necesariamente por la evaluación de la condición territorial y de los lineamientos que a ese respecto disponga la autoridad competente.

En Bogotá, la Secretaría Distrital de Salud expidió la circular 029 del 29 de abril de 2020, así como la circular 036 del 12 de mayo de 2020. En ellas se dispusieron las recomendaciones para la organización operativa de servicios ambulatorios en el contexto de la emergencia por COVID-19. Se concluyó que la prestación del servicio de salud, en el marco de la emergencia sanitaria, está asociada a un dinamismo condicionado por la evolución de la pandemia a nivel territorial y que, a medida que varíen las circunstancias, se modificarán también los lineamientos. Esto demanda un esfuerzo constante de actualización y coordinación por parte de los actores involucrados.

\section{ETAPA OPERATIVA}

\section{Preparación del personal administrativo y asistencial}

Se estimaron factores de riesgo individuales al momento de clasificar al personal administrativo y asistencial disponible para la atención presencial durante la pandemia. Además, se realizó una división en 2 grupos para la prestación rotativa de los servicios de endoscopia cada dos semanas. Se efectuaron acuerdos y talleres virtuales para la adecuación, la postura y el retiro de los EPP, y se adquirieron todos los EPP suficientes para garantizar al menos 4 meses de funcionamiento.

Asimismo, se adaptaron, modificaron y crearon algunos documentos y procedimientos necesarios para asegurar

Tabla 1. Documentos adaptados, modificados o creados para el inicio de actividad en época de pandemia*

\section{Cartilla de capacitación COVID-19 para pacientes \\ Consentimiento informado para procedimientos endoscópicos (endoscopia, colonoscopia, polipectomía) \\ Consentimiento informado para consulta presencial \\ Consentimiento informado para teleconsulta \\ Consentimiento informado para sedación \\ Consentimiento informado para acompañante \\ Declaración de estado de salud y riesgo de COVID-19 para pacientes, acompañantes, proveedores, clientes, personal administrativo y asistencial}

Sistema de gestión y seguridad en el trabajo

Hoja de ruta y verificación de procesos

Manual de bioseguridad COVID-19, lista de chequeo y medidas de contingencia

Nota de sedación COVID-19

Nota para consulta presencial y procedimientos

Procedimiento y lista de chequeo para agendamiento de procedimientos endoscópicos en pacientes con indicación semiurgente y prioritaria

Procedimiento y lista de chequeo para agendamiento de consulta presencial en época de pandemia

Procedimiento de sedación COVID-19

"Lista de documentos institucionales de Emdiagnóstica S.A.S., que se sometieron a adaptación, modificación o edición para ajustarse al escenario de COVID-19. Se detalla la información brindada a los pacientes, los formatos de consentimiento informado, los documentos organizacionales de seguridad y salud en el trabajo, los protocolos creados para guiar la atención médica, el formato de información mínima consignada en las notas clínicas de gastroenterología y anestesiología, las listas de chequeo y las hojas de ruta. COVID-19: enfermedad por coronavirus 2019 (Coronavirus Disease 2019) 
1. ¿Padece alguna enfermedad pulmonar crónica severa (asma, EPOC) o sufre de hipertensión arterial, insuficiencia renal crónica 0 enfermedades del corazón serias (incluidas infartos, enfermedad o reemplazos valvulares cardíacos) o tiene un cardio desfibrilador implantado? ¿Tiene marcapasos? ¿Padece alteraciones conocidas serias del sistema inmune?

2. ¿Usted, o alguien con quien vive, en las últimas 2 semanas ha tenido fiebre de $38^{\circ} \mathrm{C}$ o más, asociada a dolor de cabeza, tos seca, dificultad respiratoria, dolor de garganta, reducción o pérdida del olfato o el gusto (capacidad de percibir sabores) o ha presentado diarrea de reciente aparición?

3. ¿Usted, o alguien con quien vive, tiene un diagnóstico de infección por COVID-19 o ha estado en contacto con alguien en quien se esté sospechando o haya sido diagnosticado con este virus?

4. ¿Usted, o alguien con quien vive, ha estado recientemente fuera del país o en contacto con viajeros provenientes del exterior?

5. En caso de ser parte del personal de asistencia en salud, ¿tiene sospecha de haber estado en contacto no protegido con un paciente de COVID-19?

6. ¿Está dispuesto a utilizar de manera completa y adecuada todos los elementos de protección personal requeridos, los cuales serán suministrados durante su estadía en Emdiagnóstica S.A.S.?

7. Confirma que, en lo que es de su conocimiento, hasta la fecha de hoy no tiene diagnóstico de infección por COVID-19?

Si

"Formato que se entrega a diario, al inicio de la jornada, a todo el personal de Emdiagnóstica S.A.S., para confirmar su estado actual de salud y estimar el riesgo de COVID-19. Su diligenciamiento es de obligatorio cumplimiento. Solo cuando se ha respondido por completo el cuestionario, se ha tomado la temperatura corporal del empleado, se confirma que su riesgo de COVID-19 es bajo y su estado de salud es óptimo se autoriza el ingreso a las instalaciones de la unidad. Todos los formatos se almacenan en el archivo físico de Emdiagnóstica S.A.S. COVID-19: enfermedad por coronavirus 2019 (Coronavirus Disease 2019); EPOC: enfermedad pulmonar obstructiva crónica; EPP: elementos de protección personal.

el cumplimiento de los requisitos y lograr una prestación segura de servicios para los pacientes, el personal y la comunidad (Tabla 1). De igual forma, se acordó que, al inicio de cada jornada, todo el personal realizaría una declaración de su estado de salud y de riesgo de COVID-19 (Tabla 2). Todos los pacientes serían considerados como sospechosos $y$, en consecuencia, las medidas de protección serían iguales en todos los casos.

\section{Adaptación de las instalaciones físicas de la unidad de endoscopia}

Sobre la base de las publicaciones de Repici y colaboradores (17), Cennamo y colaboradores (18) y de la Asociación Americana de Gastroenterología (American Gastroenterological Association, AGA) (10), se ajustó la planta física: se demarcaron las zonas según el grado de exposición a gotas y aerosoles y se dotó la unidad con un sistema de recambio de aire con presión negativa ( 15 ciclos cada hora) con filtros recogedores de partículas de alta eficiencia (High Efficiency Particle Arresting, HEPA).

\section{Selección de pacientes y programación de procedimientos}

La prestación del servicio de teleconsulta se instauró al decretarse el aislamiento preventivo, para lo cual se acogieron los lineamientos del Gobierno respecto a la atención no presencial. Este servicio fue utilizado para priorizar la realización de los procedimientos endoscópicos. La programación de pacientes en esta fase se apoyó en las recomendaciones nacionales e internacionales sobre priorización, según la pertinencia del estudio, el estado de salud del enfermo y el comportamiento local de la curva de infección de COVID-19, junto con la adaptación de las recomendaciones de la Sociedad Asiática del Pacífico para la Endoscopia Digestiva (Asian Pacific Society for Digestive Endoscopy, APSDE), para una unidad de endoscopia ambulatoria (15) (Tabla 3), y las sugerencias de la AGA (10) en relación a las 8 semanas como límite para la realización de procedimientos sensibles al tiempo.

Así, la programación de los procedimientos se realizó fundamentada en el criterio del gastroenterólogo, en la proba- 
Tabla 3. Realización de procedimientos endoscópicos durante la pandemia por SARS-CoV-2

\begin{tabular}{|c|c|c|c|}
\hline \multicolumn{4}{|c|}{ Servicios de endoscopia durante la pandemia por COVID-19 con disponibilidad de EPP } \\
\hline $\begin{array}{l}\text { Comportamiento de } \\
\text { COVID-19 en la comunidad }\end{array}$ & $\begin{array}{c}\text { Oferta de servicios de } \\
\text { endoscopia }\end{array}$ & Estrategia & $\begin{array}{l}\text { Tiempo promedio } \\
\text { de programación } \\
\text { por sala }\end{array}$ \\
\hline $\begin{array}{l}\text { Incremento exponencial de } \\
\text { nuevos casos }\end{array}$ & Ninguno & & \\
\hline $\begin{array}{l}\text { Incremento rápido (no } \\
\text { exponencial) de casos } \\
\text { nuevos }\end{array}$ & $\begin{array}{l}\text { Realizar procedimientos } \\
\text { sensibles al tiempo, posponer } \\
\text { endoscopias electivas }\end{array}$ & Priorización 1,2 y 3 & Cada $1 \mathrm{~h}$ \\
\hline $\begin{array}{l}\text { Tendencia a la baja en casos } \\
\text { nuevos }\end{array}$ & $\begin{array}{l}\text { Realizar procedimientos } \\
\text { sensibles al tiempo, iniciar } \\
\text { procedimientos electivos }\end{array}$ & $\begin{array}{l}\text { Priorización } 1,2 \text { y } 3 . \\
\text { Diagnósticos en pacientes con factores de riesgo. } \\
\text { Tamizaje alto riesgo. } \\
\text { Seguimiento de cáncer. } \\
\text { Los necesarios para tomar conductas por otras especialidades. }\end{array}$ & Cada 40 min \\
\hline $\begin{array}{l}\text { No casos nuevos } \\
\text { diagnosticados en las últimas } \\
2 \text { semanas }\end{array}$ & Normalización & $\begin{array}{l}\text { Adicionar procedimientos diagnósticos necesarios sin factores } \\
\text { de riesgo. } \\
\text { Seguimiento de lesiones. } \\
\text { Tamizaje del riesgo promedio. }\end{array}$ & $30 \mathrm{~min}$ \\
\hline
\end{tabular}

"Estrategias utilizadas para definir la programación y realización de procedimientos endoscópicos diagnósticos durante la pandemia de COVID-19, con disponibilidad completa de EPP, y considerando el comportamiento de la curva epidemiológica local, sobre la base de las recomendaciones de la AGA (10) y la APSDE (15). COVID-19: enfermedad por coronavirus 2019 (Coronavirus Disease 2019); EPP: elementos de protección personal.

bilidad diagnóstica de cáncer y la presencia de síntomas de alarma y en el balance riesgo/beneficio en la situación de pandemia. Se establecieron 3 grupos: prioridad 1 (aquellos procedimientos que a juicio del gastroenterólogo requerían ser realizados en las dos siguientes semanas), prioridad 2 (para ser realizados a las semanas 3 y 4 ) y prioridad 3 (para efectuarse entre las semanas 5 y 8 ) (Figura 1). A todos los pacientes se les dieron recomendaciones sobre síntomas de alarma por los cuales sería necesario adelantar el procedimiento.

Un día antes de la asignación del procedimiento y de la confirmación de la cita, el personal de programación verificó de nuevo el riesgo de COVID-19. Entre tanto, en pacientes remitidos, se aplicó el procedimiento y se hizo lista de chequeo para el agendamiento de pacientes priorizados (Tabla 4). Cuando alguna de las respuestas dadas por el paciente coincidió con una señal de alerta, este se agendó para una teleconsulta, en la cual, además de definir la indicación y el momento del procedimiento, se estableció el nivel de atención en el contexto COVID-19.

Para ello estuvo disponible la escala MeNTS-ENDOGI (Figura 2). Esta fue adaptada de la publicación de Prachand y colaboradores (19), a partir de ajustes en los criterios evaluados, a fin de poder aplicarla en el escenario de procedimientos endoscópicos y lograr objetivar la decisión. Se estableció la seguridad del ambiente ambulatorio para un valor de hasta 45 puntos. En valores más altos, la recomendación fue remitir al paciente a la realización del procedimiento a un nivel intrahospitalario.

\section{ETAPA OPERATIVA}

\section{Dotación de equipos para protección personal y bioseguridad}

Se siguieron los lineamientos del American College of Surgeons (20), de la AGA (10), del Ministerio de Salud y de la Asociación Colombiana de Infectología (11) para establecer el uso de EPP según las áreas de riesgo y actividad, incluidos los pacientes (Tabla 5). Entre tanto, para la postura y el retiro seguros de los EPP se recibió una capacitación virtual y se dispuso de una infografía en cada sala. Solo está permitido el uso de los EPP entregados por la institución.

\section{Protocolo para sedación}

Todos los procedimientos endoscópicos en la unidad se realizan bajo sedación administrada por anestesiólogos. Antes de la pandemia, era usual mantener a los pacientes en un nivel II de sedación, según la clasificación de la Practice Guidelines for Sedation and Analgesia by non-Anesthesiologist (21). Sin embargo, por el requerimiento de un mayor grado de profundidad, necesario para lograr el paso seguro del 


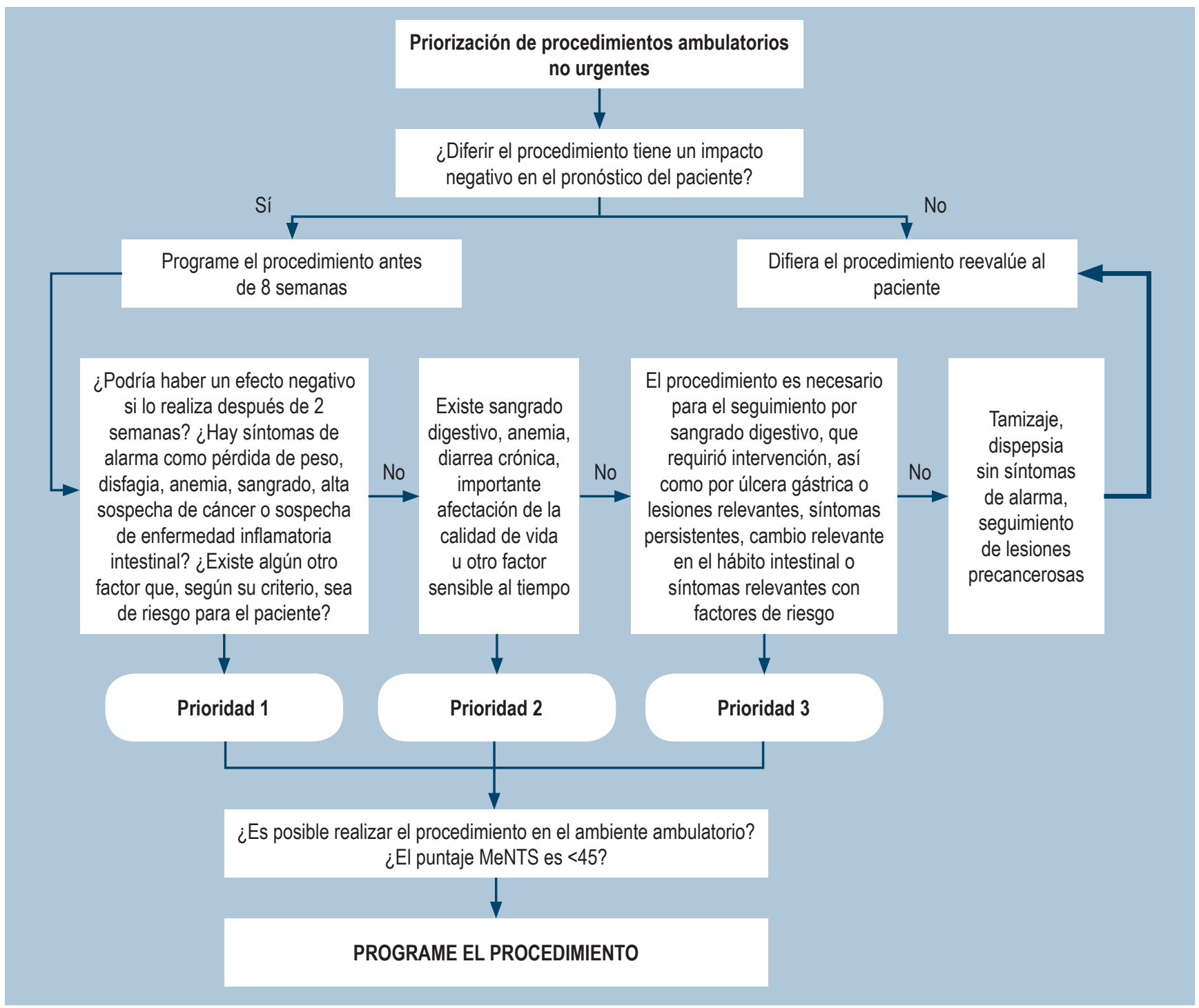

Figura 1. Flujograma para la priorización de procedimientos endoscópicos durante la fase de mitigación y ascenso de la curva COVID-19. La decisión de programar o no un procedimiento recae en la juiciosa evaluación clínica y en la determinación de la pertinencia del estudio durante el período de pandemia. Además, se toma en consideración la estimación del puntaje MeNTS-ENDOGI. MeNTS: Medically Necessary Time-Sensitive

cricofaríngeo sin reflejo tusígeno, se decidió administrar los medicamentos convencionalmente utilizados (remifentanilo y propofol) hasta conseguir dicho objetivo.

Asimismo, para disminuir los períodos de apnea prolongados con desaturación e hipoxemia, se estableció de manera obligatoria que todos los pacientes recibirían preoxigenación rutinaria por $3 \mathrm{~min}$ con oxígeno por cánula nasal a 2-3 L/min (debajo de la barrera de aislamiento), con inspiraciones profundas. En caso de que la apnea del paciente se acompañara de desaturación (saturación de oxígeno por pulsioximetría $\left.\left[\mathrm{SpO}_{2}\right]<90 \%\right)$, se definió como plan de acción incrementar el flujo de oxígeno hasta $5 \mathrm{~L} / \mathrm{min}$ y realizar maniobras para la permeabilización de la vía aérea.
Si a pesar de esto la desaturación persistía, la indicación fue suspender el procedimiento endoscópico y administrar oxígeno por máscara facial con técnica de sello hermético a cuatro manos, a fin de evitar al máximo la intubación endotraqueal o la ventilación a presión positiva. Se adoptó una lista de chequeo de complicaciones asociadas a la sedación (22).

\section{Procedimientos endoscópicos}

Los pacientes se programaron de manera alternada en cada una de las tres salas disponibles, lo que permitióla ventilación, la limpieza y la desinfección de cada zona, previas al ingreso del siguiente paciente. De esta manera, a pesar de contar con 
Tabla 4. Proceso y lista de chequeo para el agendamiento de procedimientos endoscópicos en pacientes priorizados*

1. ¿Está de acuerdo con que agendemos el procedimiento a pesar de la pandemia de COVID-19? Deje nota en la historia clínica y agende para teleconsulta

2. ¿Ha presentado una pérdida alarmante de peso, anemia o cambios significativos en los hábitos defecatorios (diarrea/ estreñimiento)? ¿Tiene dificultad para deglutir (tragar los alimentos), ha presentado sangrado digestivo o sabe que su médico tiene una alta sospecha de cáncer en su situación particular?

3. ¿Se encuentra anticoagulado? Si la respuesta es Sí, continúe con la pregunta 4; de lo contrario, pase a la 7 terapia puente?

5. ¿Es mayor de 70 años?

6. ¿Vive en un asilo o en un lugar de atención para pacientes de la tercera edad?

7. ¿Padece alguna enfermedad pulmonar crónica severa (asma, EPOC) o sufre de hipertensión arterial controlada inadecuadamente, insuficiencia renal crónica o enfermedades del corazón serias (incluidas infartos, enfermedad o reemplazos valvulares cardiacos) 0 tiene un cardio desfibrilador implantado? ¿Tiene marcapasos? ¿Padece alteraciones conocidas serias del sistema inmune?

8. ¿Tiene obesidad, apnea del sueño o es diabético? Si la respuesta es Sí, agende una teleconsulta

9. ¿Se encuentra en embarazo?

10. ¿Es capaz de caminar 3 cuadras o subir dos pisos sin que le falte el aire?

11. ¿Usted, o alguien con quien vive, en las últimas 2 semanas ha tenido fiebre de $38^{\circ} \mathrm{C}$ o más, asociada a dolor de cabeza, tos seca, dificultad respiratoria, dolor de garganta, reducción o pérdida del olfato 0 el gusto (capacidad de percibir sabores) 0 ha presentado diarrea de reciente aparición?

12. ¿Usted, o alguien con quien vive, tiene diagnóstico de infección por COVID-19 o ha estado en contacto con alguien de quien se sospecha o ha sido diagnosticado con este virus?

13. ¿Usted, o alguien con quien vive, ha estado fuera del país o en contacto durante las últimas 2 semanas con viajeros provenientes del exterior?

"Lista de preguntas que se realizan al paciente, por vía telefónica, desde la central de agenda de citas de Emdiagnóstica S.A.S., para definir si se continúa directamente con la programación del procedimiento o, en su defecto (áreas sombreadas en azul), se deriva a la valoración especializada por gastroenterología en la modalidad de teleconsulta. Esto, a fin de individualizar y definir la conducta. Este formato funciona como prueba de tamizaje para filtrar a los pacientes según su riesgo y optimizar la programación de las citas. COVID-19: enfermedad por coronavirus 2019 (Coronavirus Disease 2019); EPOC: enfermedad pulmonar obstructiva crónica.

presión negativa, cada sala se usa nuevamente después de $1 \mathrm{~h}$ y así se utiliza, inicialmente, el $30 \%$ de la capacidad instalada. De otro lado, se usa una protección con barrera plástica para endoscopia (23) y una sábana fenestrada para colonoscopia. En todos los casos, se diligencia el registro de indicadores de calidad para endoscopia y colonoscopia en la hoja de ruta $y$ verificación de procesos de manera usual y se realiza la resección de lesiones encontradas dependiendo de la caracterización de cada lesión. El personal asistencial cambia la bata antifluido externa en cada paciente.

\section{ETAPA POSOPERATIVA}

\section{Seguimiento y retroalimentación}

Todos los pacientes son seguidos mediante una encuesta de satisfacción después del procedimiento; también para la verificación de complicaciones luego de la polipectomía, entre las $12-48$ h, y a los 7 y 14 días. Además, se les pregunta sobre su grado de satisfacción y su sensación de seguridad mientras permanecieron en la unidad (Tabla 6). 
A. Modelo de la escala

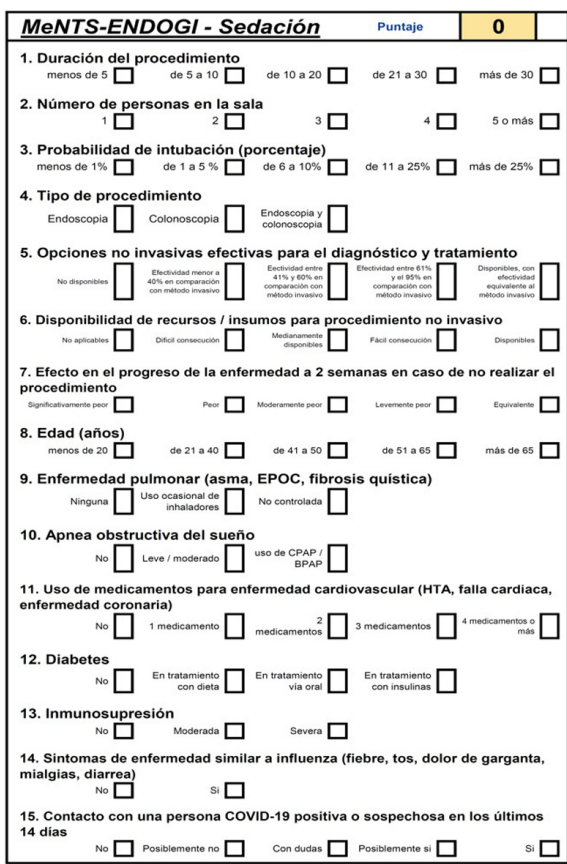

B. Procedimiento programable en ambiente ambulatorio

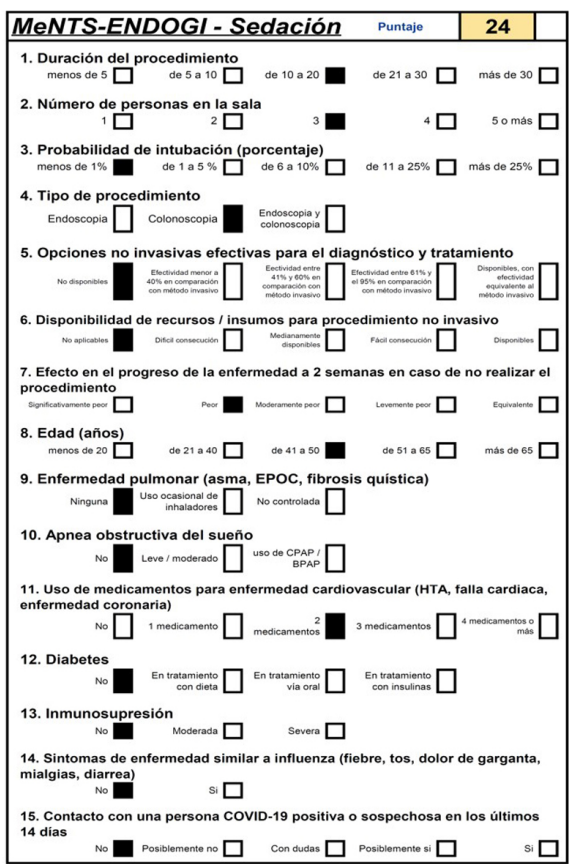

C. Procedimiento no programable en el ambiente ambulatorio

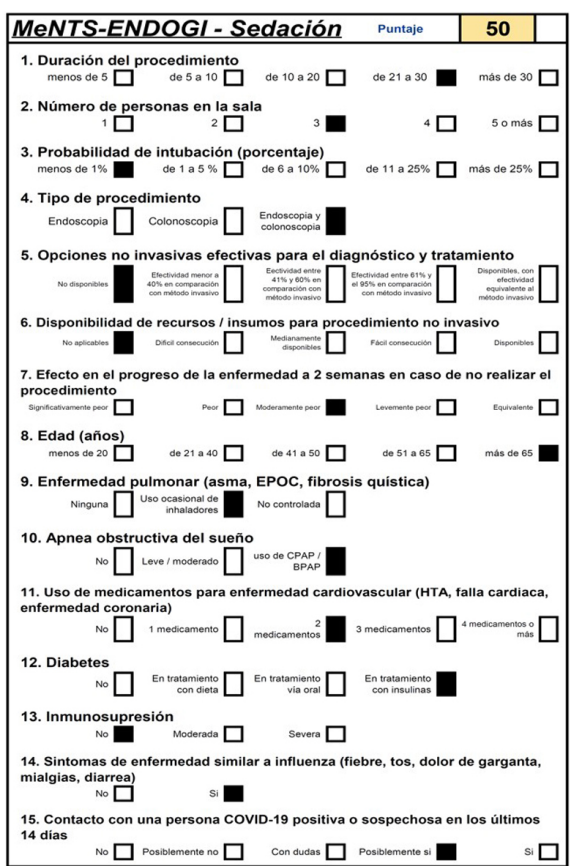

Figura 2. Escala MeNTS-ENDOGI. Estos son esquemas representativos de la escala, aplicados por Emdiagnóstica S.A.S. Dicha escala es una adaptación del modelo MeNTS original de Prachand y colaboradores. El panel A muestra el formato en blanco, antes de su diligenciamiento. El panel B plasma el resultado de aplicar la escala MeNTS-ENDOGI en un paciente con bajo riesgo de complicaciones y de diseminación de COVID-19, el cual puede ser programado para realizar su procedimiento de manera ambulatoria. El panel C corresponde a un paciente que, por sus factores de riesgo (clínicos y de diseminación de infección COVID-19), no puede agendarse como procedimiento ambulatorio. Los dos casos descritos son escenarios simulados y no representan información de pacientes reales. Emdiagnóstica S.A.S. asumió el punto de corte de 45 puntos para definir si el procedimiento se podía efectuar en un entorno ambulatorio $u$ hospitalario.

\section{DISCUSIÓN}

El confinamiento obligatorio logró contener el crecimiento exponencial de la pandemia en el país, lo cual dio tiempo para evitar el colapso de las UCI; sin embargo, también generó la acumulación de un número progresivo de procedimientos no urgentes, pero sensibles al tiempo, que no pudieron realizarse por causa del cierre de las unidades ambulatorias de endoscopia.

Por tanto, dichos procedimientos requirieron ser programados en la ventana de tiempo actual de atención, a fin de optimizar al máximo los recursos disponibles en las unidades de endoscopia preparadas para una atención segura, y durante un período incierto, que depende especialmente del porcentaje de ocupación de UCI.

En Colombia, se dio una condición diferente a la de países con curvas exponenciales, que no tuvieron este período de contención. La situación actual de pandemia ha requerido de liderazgo y trabajo en equipo en las empresas prestadoras de servicios de salud, para proponer soluciones y evaluar propuestas aplicables a un escenario diferente al que tuvieron otros países, con un comportamiento epidemiológico distinto.

Las adaptaciones realizadas en nuestra unidad han permitido reiniciar una atención con el 30\% de la capacidad instalada antes del pico epidemiológico, planeando incrementos progresivos según el descenso de la curva, siendo indispensable determinar criterios para la utilización apropiada de la capacidad instalada, del talento humano y de los insumos siempre en consonancia con la situación local, para así responder la pregunta de ¿cómo reiniciar?”

$\mathrm{Al}$ presentar este documento como experiencia de trabajo, entendemos que el escenario de la COVID-19 es dinámico e incluso impredecible. Con la propuesta de programación por prioridades en este período de ventana pretendemos responder la cuestión sobre a quiénes programar, con la intención de optimizar el recurso diagnóstico y llevar al tratamiento oportuno para impactar directamente en el pronóstico de los pacientes.

Los resultados obtenidos mediante el registro de procedimientos, el seguimiento a los pacientes, las medidas para 
Tabla 5. EPP de acuerdo con el área de exposición y el rol desempeñado*

\begin{tabular}{ll}
\hline \multicolumn{1}{|c|}{$\begin{array}{c}\text { Área de exposición / rol } \\
\text { desempeñado }\end{array}$} & \multicolumn{1}{c|}{ EPP } \\
\hline $\begin{array}{l}\text { Área de procedimientos } \\
\text { (personal asistencial: } \\
\text { gastroenterólogos, } \\
\text { anestesiólogos, auxiliares } \\
\text { de enfermería) }\end{array}$ & $\begin{array}{l}\text { Respiradores N95 } \\
\text { Gafas } \\
\text { Máscara facial } \\
\text { Gorro } \\
\text { Bata antifluido doble (la bata externa se } \\
\text { cambia para cada nuevo paciente) }\end{array}$ \\
$\begin{array}{ll}\text { Doble guante } \\
\text { Polainas }\end{array}$ \\
$\begin{array}{l}\text { Área administrativa } \\
\text { (secretarias, recepcionista, } \\
\text { asistente administrativa) }\end{array}$ & $\begin{array}{l}\text { Tapabocas y máscara facial } \\
\text { Pacientes en área de } \\
\text { procedimientos }\end{array}$ \\
\end{tabular}

*Lista de EPP clasificados según el nivel de riesgo de exposición. Para la asignación de riesgo, se consideró el área de trabajo (a fin de determinar el grado de exposición a gotas y aerosoles) y el rol desempeñado por cada uno de los empleados. Para la elaboración de esta lista se utilizó la guía del American College of Surgeons (20). EPP: elementos de protección personal.

disminuir el contagio y los diagnósticos logrados son temas que deben considerarse en estudios posteriores. El reinicio de actividades también ha mostrado cambios en la práctica de la especialidad, que implican grandes retos para el funcionamiento sostenible de las unidades de endoscopia.

De igual forma, los aspectos económicos (mayores costos con menor número de procedimientos) podrían llegar a amenazar la estabilidad de las instituciones prestadoras de salud (IPS) y prestadores independientes. Filtrar, priorizar y ponderar los casos basados en el criterio clínico, a partir de una justa planeación del gasto, nunca fue tan importante dentro de un marco legal cambiante. El reto
Tabla 6. Preguntas para el seguimiento a pacientes durante la pandemia de COVID-19*

1. Posterior a la realización de su procedimiento endoscópico, ¿ha presentado fiebre, tos, dificultad para percibir olores 0 sabores 0 dificultad al respirar?

En caso de que la respuesta sea positiva, notifique de inmediato a la dirección médica

2. Posterior a la realización del procedimiento endoscópico, ¿su salud se ha deteriorado?

En caso de que la respuesta sea positiva, notifique de inmediato a la dirección médica.

3. ¿Ha aparecido algún síntoma inusual posterior a la realización de su procedimiento endoscópico?

4. En caso de que la respuesta a la pregunta 3 sea afirmativa, indique cuál síntoma ha presentado y notifique de inmediato a la dirección médica.

5. ¿Cuál es la percepción de seguridad que tuvo durante la realización del procedimiento? El procedimiento endoscópico le pareció:
A). Muy inseguro
B). Inseguro
C). Seguro
D). Muy seguro

6. ¿Tiene alguna recomendación u observación para realizar?

*Cuestionario para el seguimiento telefónico a los pacientes, en quienes se realizó un estudio endoscópico diagnóstico en Emdiagnóstica S.A.S. Los datos obtenidos se utilizan para evaluar la posibilidad de casos de COVID-19 que se hayan pasado por alto, debido al período de ventana (enfermos asintomáticos), y para estimar el grado de satisfacción de los pacientes con la atención recibida. Se evalúa también el nivel de seguridad percibido por el paciente durante su estancia en Emdiagnóstica S.A.S.

ahora es adaptarnos para seguir prestando servicios sostenibles y de calidad.

\section{Agradecimientos}

A todo el personal administrativo y asistencial de Emdiagnóstica S.A.S., un grupo maravilloso de gastroenterólogos, anestesiólogos y auxiliares de enfermería que se esfuerzan en seguir las recomendaciones dentro de un sistema de gestión planeado para disminuir riesgos y prestar una atención segura y de calidad.

\section{REFERENCIAS}

1. World Health Organization [internet]. WHO DirectorGeneral's opening remarks at the media briefing on COVID-19 - 11 March 2020: Coronavirus disease
(COVID-2019) situation report-50 [actualizada el $11 \mathrm{de}$ marzo de 2020]. Disponible en: https://www.who.int/dg/ 
speeches/detail/who-director-general-s-opening-remarksat-the-media-briefing-on-covid-19---11-march-2020

2. Ministerio de Salud y Protección Social [internet]. Lineamientos de prevención del contagio por COVID-19 y atención en salud para las personas con discapacidad, sus familias, las personas cuidadoras y actores del sector salud. Bogotá: marzo de 2020. Disponible en: https://www. minsalud.gov.co/sites/rid/Lists/BibliotecaDigital/RIDE/ DE/PS/asif13-personas-con-discapacidad.covid-19.pdf

3. Ministerio de Salud y Protección Social [internet]. Lineamientos para el manejo clínico de pacientes con infección por nuevo coronavirus COVID-19. Bogotá: julio de 2020. Disponible en: https: / www.minsalud.gov.co/ Ministerio/Institucional/Procesos\%20y\%20procedimientos/PSSS03.pdf

4. Ministerio de Salud y Protección Social [internet]. Colombia confirma su primer caso de COVID-19: boletín de prensa [actualizada el 6 de marzo de 2020]. Disponible en: https://www.minsalud.gov.co/Paginas/Colombiaconfirma-su-primer-caso-de-COVID-19.aspx

5. Decreto número 636 de 2020. Por el cual se imparten instrucciones en virtud de la emergencia generada por la pandemia del coronavirus COVID-19, y el mantenimiento del orden público (6 de mayo 2020). Ministerio del Interior de la República de Colombia.

6. Presidencia de la República de Colombia [internet]. Aislamiento preventivo obligatorio [consultada el 25 de agosto de 2020]. Disponible en: https://id.presidencia.gov. co/especiales/200323-Aislamiento-Preventivo-Obligatorio/index.html

7. Tse F, Borgaonkar M, Leontiadis GI. COVID-19: Advice from the Canadian Association of Gastroenterology for Endoscopy Facilities, as of March 16, 2020. J Can Assoc Gastroenterol. 2020;3(3):147-149. http://doi.org/10.1093/jcag/gwaa012

8. American Gastroenterological Association [internet]. Joint GI society message: COVID-19 clinical insights for our community of gastroenterologists and gastroenterology care providers. 2020 [actualizada el 16 de marzo de 2020]. Disponible en: https://gastro.org/press-releases/ joint-gi-society-message-covid-19-clinical-insights-for-ourcommunity-of-gastroenterologists-and-gastroenterologycare-providers/

9. Thompson CC, Shen L, Lee LS. COVID-19 in endoscopy: Time to do more? Gastrointest Endosc. 2020;92(2):435-439. http://doi.org/10.1016/j.gie.2020.03.3848

10. Sultan S, Lim JK, Altayar O, Davitkov P, Feuerstein JD, Siddique SM, Falck-Ytter Y, El-Serag HB; AGA Institute. AGA Rapid Recommendations for Gastrointestinal Procedures During the COVID-19 Pandemic. Gastroenterology. 2020;159(2):739-758.e4. http://doi.org/10.1053/j.gastro.2020.03.072

11. Consenso colombiano de atención, diagnóstico y manejo de la infección por SARS-COV-2/COVID-19 en establecimientos de atención de la salud: Recomendaciones basadas en consenso de expertos e informadas en la evidencia. Infectio. 2020;24(3 Suppl 2).

12. Sociedad Española de Endoscopia Digestiva (SEED) [internet]. Recomendaciones de la SEED: protección en unidades de endoscopia frente al COVID-19. 2020 [actualizada el 13 de marzo de 2020]. Disponible en: https://www.wseed.org/ josepr23/sociedades/SEEDv3/ images/site/guia_clinica/2020/RecomendacionesSEED_ UnidadesEndoscopia_COVID19_v1.pdf

13. Beilenhoff U, Biering $\bar{H}$, Blum R, Brljak J, Cimbro M, Dumonceau JM, Hassan C, Jung M, Kampf B, Neumann C, Pietsch M, Pineau L, Ponchon T, Rejchrt S, Rey JF, Schmidt V, Tillett J, van Hooft JE. Reprocessing of flexible endoscopes and endoscopic accessories used in gastrointestinal endoscopy: Position Statement of the European Society of Gastrointestinal Endoscopy (ESGE) and European Society of Gastroenterology Nurses and Associates (ESGENA)-Update 2018. Endoscopy. 2018;50(12):1205-1234. http://doi.org/10.1055/a-0759-1629

14. Aguila EJT, Lontok MAD, Francisco CPD. Follow Your Gut: Challenges in Nutritional Therapy During the COVID-19 Pandemic. Clin Gastroenterol Hepatol. 2020;S1542-3565(20)30753-9. http://doi.org/10.1016/j.cgh.2020.05.046

15. Chiu PWY, Ng SC, Inoue H, Reddy DN, Ling Hu E, Cho JY, Ho LK, Hewett DG, Chiu HM, Rerknimitr R, Wang HP, Ho SH, Seo DW, Goh KL, Tajiri H, Kitano S, Chan FKL. Practice of endoscopy during COVID-19 pandemic: position statements of the Asian Pacific Society for Digestive Endoscopy (APSDE-COVID statements). Gut. 2020;69(6):991-996. http://doi.org/10.1136/gutjnl-2020-321185

16. American Society for Gastrointestinal Endoscopy [internet]. Guidance for resuming GI endoscopy and practice operations after the COVID-19 pandemic. 2020 [actualizada el 28 de abril de 2020]. Disponible en: https: / /www. asge.org/docs/default-source/default-document-library/ asge-guidance-for-reopeningl_4-28-2020.pdf

17. Repici A, Maselli R, Colombo M, Gabbiadini R, Spadaccini M, Anderloni A, Carrara S, Fugazza A, Di Leo M, Galtieri PA, Pellegatta G, Ferrara EC, Azzolini E, Lagioia M. Coronavirus (COVID-19) outbreak: what the department of endoscopy should know. Gastrointest Endosc. 2020;92(1):192-197. http://doi.org/10.1016/j.gie.2020.03.019

18. Cennamo V, Bassi M, Landi S, Apolito P, Ghersi S, Dabizzi E, Polifemo AM, Gizzi G, Guicciardi S, Indelicato G, Cascone C, Tovoli D, Tumietto F, Viale P, Jovine E, Repici A. Redesign of a GI endoscopy unit during the COVID-19 emergency: A practical model. Dig Liver Dis. 2020. http://doi.org/10.1016/j.dld.2020.05.007

19. Prachand VN, Milner R, Angelos P, Posner MC, Fung JJ, Agrawal N, Jeevanandam V, Matthews JB. Medically Necessary, Time-Sensitive Procedures: Scoring System to 
Ethically and Efficiently Manage Resource Scarcity and Provider Risk During the COVID-19 Pandemic. J Am Coll Surg. 2020;231(2):281-288.

http://doi.org/10.1016/j.jamcollsurg.2020.04.011

20. American College of Surgeons [internet]. Personal Protective Equipment (PPE): ASA and APSF Joint Statement on Perioperative Testing for the COVID-19 Virus. 2020 [actualizada el 1 junio de 2020]. Disponible en: https://www.asahq.org/about-asa/newsroom/ news-releases/2020/04/asa-and-apsf-joint-statement-onperioperative-testing-for-the-covid-19-virus

21. American Society of Anesthesiologists Task Force on Sedation and Analgesia by Non-Anesthesiologists. Practice guidelines for sedation and analgesia by non-anesthesiolo- gists. Anesthesiology. 2002;96(4):1004-1017. http://doi.org/10.1097/00000542-200204000-00031

22. Roback MG, Green SM, Andolfatto G, Leroy PL, Mason KP. Tracking and Reporting Outcomes Of Procedural Sedation (TROOPS): Standardized Quality Improvement and Research Tools from the International Committee for the Advancement of Procedural Sedation. Br J Anaesth. $2018 ; 120(1): 164-172$. http://doi.org/10.1016/j.bja.2017.08.004

23. Luis S, Margarita H, Javier P, Daniela S. New protection barrier for endoscopic procedures in the era of pandemic COVID-19. VideoGIE. 2020;10.1016/j.vgie.2020.05.006. http://doi.org/10.1016/j.vgie.2020.05.006 